This item was submitted to Loughborough's Research Repository by the author.

Items in Figshare are protected by copyright, with all rights reserved, unless otherwise indicated.

\title{
Patch antenna directivity analysis using an equi-area method
}

PLEASE CITE THE PUBLISHED VERSION

PUBLISHER

(C) IEEE

VERSION

VoR (Version of Record)

LICENCE

CC BY-NC-ND 4.0

REPOSITORY RECORD

Ullah, Sadiq, James A. Flint, and R.D. Seager. 2019. "Patch Antenna Directivity Analysis Using an Equi-area Method". figshare. https://hdl.handle.net/2134/6167. 
This item was submitted to Loughborough's Institutional Repository (https://dspace.lboro.ac.uk/) by the author and is made available under the following Creative Commons Licence conditions.

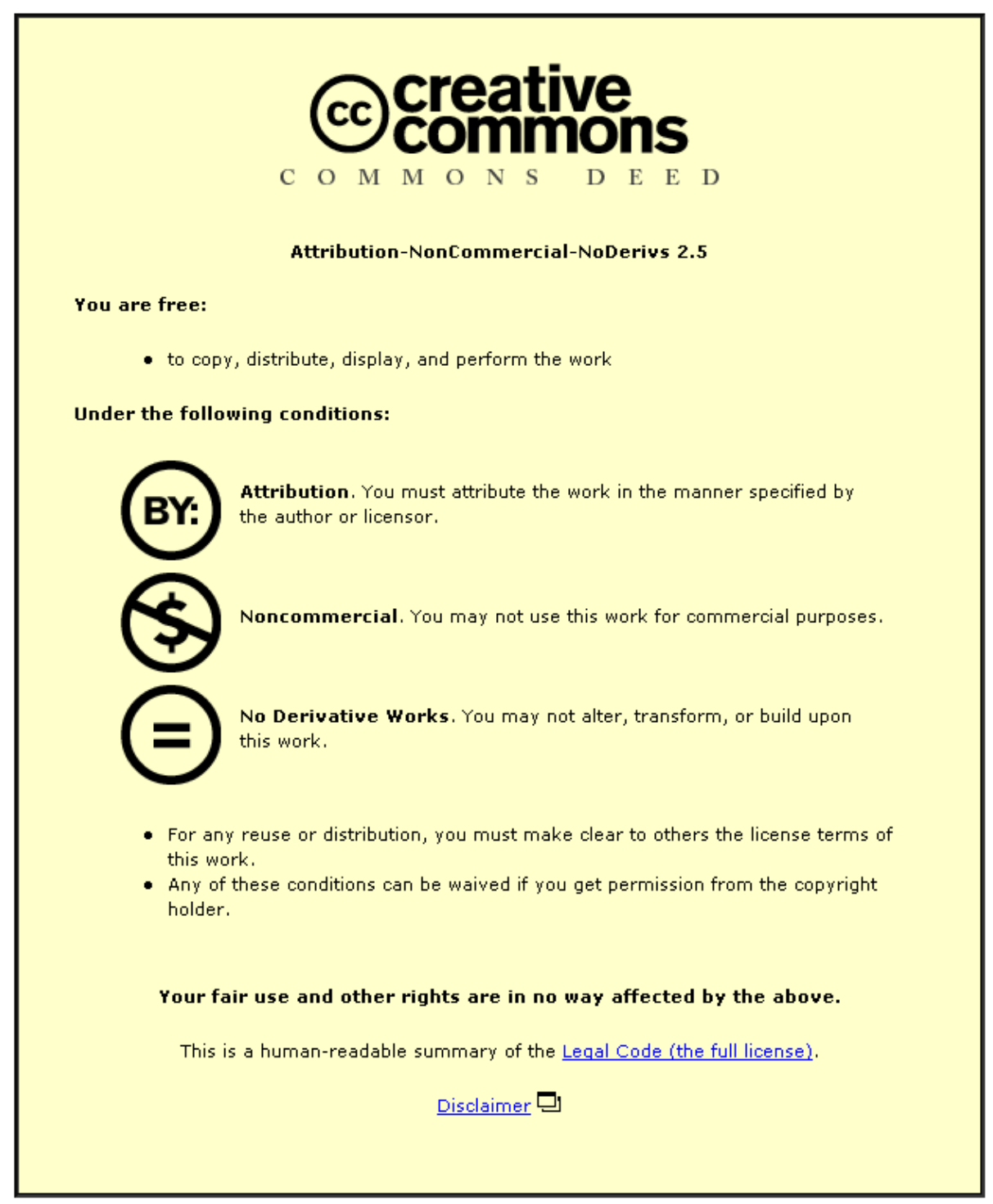

For the full text of this licence, please go to: http://creativecommons.org/licenses/by-nc-nd/2.5/ 


\title{
PATCH ANTENNA DIRECTIVITY ANALYSIS USING AN EQUI-AREA METHOD
}

\author{
S. Ullah, J. A. Flint, R. D. Seager \\ Department of Electronic \& Electrical Engineering, Loughborough University, UK. \\ E-Mail (s): \{S. Ullah, J.A. Flint, R. D. Seager\}@lboro.ac.uk
}

\begin{abstract}
The widespread exploitation of mobile cellular phones and use of wireless devices such as PDAs, in-vehicle Global Positioning System (GPS) receivers, and the future deployment of mobile satellite digital audio and video has developed a re-energized interest in efficient and accurate measurement techniques for antennas. This paper demonstrates directivity analysis of a patch antenna using a new fast, rotation-invariant spherical near-field antenna measurement technique. The method is based on an equi-area surface partitioning algorithm. The paper evaluates the sampling technique's performance when compared with a normal spherical near-field measurement technique using equal angle sampling.
\end{abstract}

\section{Introduction}

The need to rapidly and efficiently characterize the performance of antennas system is an important demand on the measurement community. It is frequently necessary to evaluate the near field and far field characteristics of antennas both numerically and experimentally. Conventionally the antenna radiation pattern would be calculated either by Great Circle Method (GCM) [1] or the Conical Section Method (CSM) [1] both utilising an equi-angle based method. I.e. in both cases the scan is carried out by incremental changes in elevation angle $\theta$ and azimuth angle $\varphi$ in the spherical coordinate system.

The antenna radiation intensity pattern $U(\theta, \varphi)$ is used to find the total radiation power $\left(P_{\text {rad }}\right)$ of an antenna by applying the exact equation as given by Balanis [2], i.e.

$$
P_{\text {rad }}=\int_{0}^{2 \pi} \int_{0}^{\pi} U(\theta, \phi) d \Omega=\int_{0}^{2 \pi} \int_{0}^{\pi} U(\theta, \phi) \sin \theta d \theta d \phi
$$

Where $d \Omega=$ element of solid angle.

Similarly the directivity of an antenna is the ratio of its radiation intensity in a given direction to the radiation intensity averaged over all directions i.e.

$$
D=\frac{U}{U_{\text {avg }}}=\frac{U}{\left(P_{\text {rad }} / 4 \pi\right)}=\frac{4 \pi U}{P_{r a d}}
$$

These analytical integral equations are usually calculated numerically via equi-angle-based summations as given in Balanis [2] and Kraus [3]. The fundamental concept of this numerical method is the Equi-angle based distribution of the sampling points throughout the spherical surface in both azimuth and elevation axes, i.e., if,

$$
U=B_{0} U(\theta, \phi)
$$

Where, $U$ is the radiation intensity and $B_{0}$ is a constant.

$$
\Delta \theta_{i}=\frac{\pi}{L}, \Delta \phi_{j}=\frac{2 \pi}{M}, M=2 L, \theta_{i}=i \Delta \theta_{i}=\frac{i \pi}{L}, i=1,2, \ldots, L, \phi_{j}=j \Delta \phi_{j}=\frac{j 2 \pi}{M}, j=1,2, \ldots, M
$$




$$
\begin{aligned}
& P_{\text {equi-angle }}=\left(\frac{\pi}{L}\right)\left(\frac{2 \pi}{M}\right) B_{0} \sum_{i=1}^{L} \sum_{j=1}^{M} U\left(\theta_{i}, \phi_{j}\right) \sin \left(\theta_{i}\right) \\
& D_{\text {equi-angle }}=\frac{4 \pi * U_{\max }}{P_{\text {equi-angle }}}
\end{aligned}
$$

It should be noted that this method uses a set of sampling points that whilst incremental in $\varphi, \theta$, are not spaced equally on the surface of the unit sphere. Consequently the accuracy of the results will vary, depending on the alignment of the pattern of the coordinate system. Therefore it would be desirable to reduce the dependency of the measurement on the antenna alignment. This is particularly relevant when the antenna under test is beamsteered or multi-beam. There are a number of options for selecting the sampling locations on the spherical surface in a more uniform manner. One of the most promising approaches involves optimising the area on the surface so that each point is placed centrally within its own equal area segment. Leopardi [4] has obtained a mathematical procedure for sphere partitioning which is discussed in the next section

Section 2 describes the equi-area-method in detail together with Leopardi's [4] mathematical model for sphere partitioning. Patch antenna directivity using an equi-area method is discussed in Section 3. Results are presented in section 4; Section 5 concludes the paper.

\section{Equi-Area Method}

The method described in the current paper utilises the equal area based distribution of the sampling points on the spherical surface around the antenna. The recursive zonal equal area sphere partitioning algorithm [4] is applied to the patch antenna radiation pattern; i.e. the 3 dimensional unit-sphere is divided into $N$ equi-area regions. The centre points of these equi-area regions are obtained in Cartesian coordinate system for the 3 dimensional unitsphere. We convert the centre points of the equi-regions from Cartesian to spherical coordinates $(r, \theta, \varphi)$. The antenna radiation power pattern is then sampled using these spherical coordinates. Finally, the samples are summed to give the total antenna radiation power. The directivity of the antenna is computed by finding the maxima and then using the total antenna radiation power.

The equi-regions each represented by $R$, having an equal area $\mathrm{A}_{R}$ equal to the area of the sphere $(\Omega)$ divided by $N$, i.e.

$$
\mathrm{A}_{R}=\frac{\Omega}{N}
$$

The Leopardi's [4] algorithm uses the recursive method outlined below:

If $N=1$ then

There is Single region (whole sphere);

else if $d=1$ then

Divide the circle into $N$ equal segments;

else

Divide the sphere into north and south polar caps and intermediate collars:

Determine the colatitudes of polar caps;

Determine an ideal collar angle;

Determine an ideal number of collars;

Determine the actual number of collars;

Create a list of the ideal number of regions in each collar;

Create a list of the actual number of regions in each collar;

Partition each spherical collar into regions of equal area, using the recursive zonal equal area partition algorithm for dimension $d-1$;

endif 
The Leopardi's algorithm is recursive in the dimension $d$, because it uses a recursive rounding-function for calculating the actual number of regions in each collar, so that the total number of equi-regions on the spherical surface should not exceed $N$. The coordinates of the centre points of the equi-area regions are found using this algorithm. The block diagram of the equi-area method is illustrated in Fig. 1 whereas the spherical surface divided into equal areas according to Leopardi algorithm [4] is shown in Fig. 2. The antenna radiation intensity pattern is sampled at the centre points of the equi-area regions. The sampling points are shown in Fig. 2.

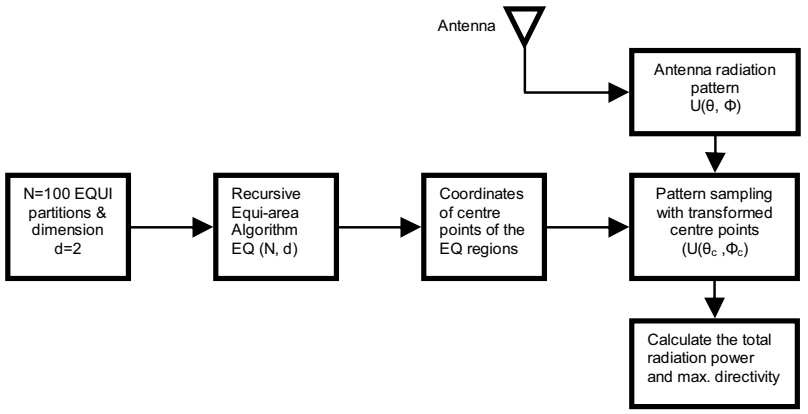

Fig. 1. Block diagram of Equi-area Method

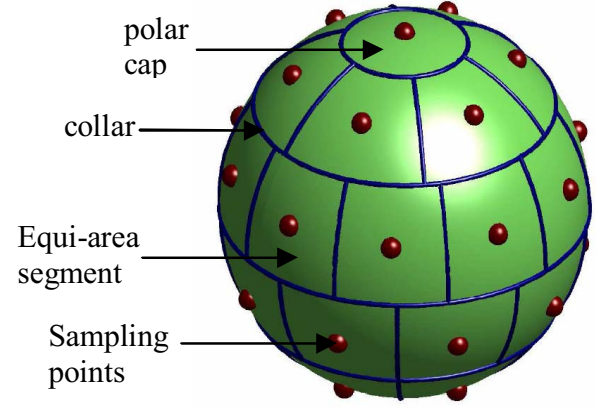

Fig. 2. Equi-Area sampling of the unit sphere [4]

Consequently if $U\left(\theta_{c, j}, \phi_{c, j}\right)$ represents the radiation intensity sampled at the center of the jth equi-region, then the total radiated power is calculated by using the equi-area method as

$$
P_{\text {equi-area }}=\mathrm{A}_{R} \sum_{j=1}^{N} U\left(\theta_{c, j}, \phi_{c, j}\right)
$$

The maximum directivity for the antenna having a maximum radiation intensity of $\left(U_{\max }\right)$ is calculated in the same manner as explained previously

$$
D_{\text {equi-area }}=\frac{U_{\max } * 4 \pi}{P_{\text {equi-area }}}
$$

\section{Patch antenna directivity calculations using equi-area algorithm}

The microstrip patch antenna of length $(L)$, width $(W)$ with a substrate of thickness $(h)$ can be represented by two radiating slots separated by the length of the patch. The electric field $(E)$ radiated by each slot at a distance $(r)$ is given by [2], and the radiation intensity $(U)$ of the patch antenna is

$$
U=|E|^{2} / 2 \eta_{0}=\left|j \frac{V_{0} e^{-j k_{0} r}}{\pi r}\left\{\sin \theta \frac{\sin \left(\frac{k_{0} W}{2} \cos \theta\right)}{\cos \theta}\right\}\right| / 2 \eta_{0}
$$

Where $V_{0}$ and $k_{0}=2 \pi / \lambda_{0}$ are the voltage due to the field and wave number in free space respectively. $\lambda_{0}$ is the free-space wavelength. $\eta_{0}$ is the free-space impedance. The value of $U_{\max }$ for patch antenna is given in [2]. The radiation power of the patch antenna using the equi-area method (i.e. $P_{\text {equi-area }}$ ) is obtained and the corresponding patch antenna directivity $\left(D_{\text {equi-area }}\right)$ is calculated 


\section{Results}

In this paper equi-area method is used to analyze the patch antenna having a resonance frequency of $10 \mathrm{GHz}$. The patch dimensions are obtained by using the transmission line model [2]. I.e. a square patch of length and width equal to half of a wavelength (i.e. $10.11 \mathrm{~mm}$ ), on a substrate of height $1.6 \mathrm{~mm}$ and dielectric constant 2.2 is obtained. First of all the exact value of the maximum directivity for the patch antenna is calculated using the analytical expression, which gives an exact value of 6.62 (i.e. $8.20 \mathrm{~dB}$ ). The equi-angle based method gives a maximum directivity of $6.24(7.95 \mathrm{~dB})$. On the other hand the equi-area based method gives maximum directivity of $6.65(8.23 \mathrm{~dB})$ for fewer sampling points which is stable throughout the rotation cycle. It is worth noting that when the antenna radiation pattern is rotated relative to the coordinate system, a periodic deviation in the directivity is seen for the equi-angle method as shown in Fig. 3 and Fig. 4. The asymmetry in the maximum directivity curve about the 180 degrees point for the equi-area method, is due to the presence of the back lobes in the radiation pattern.

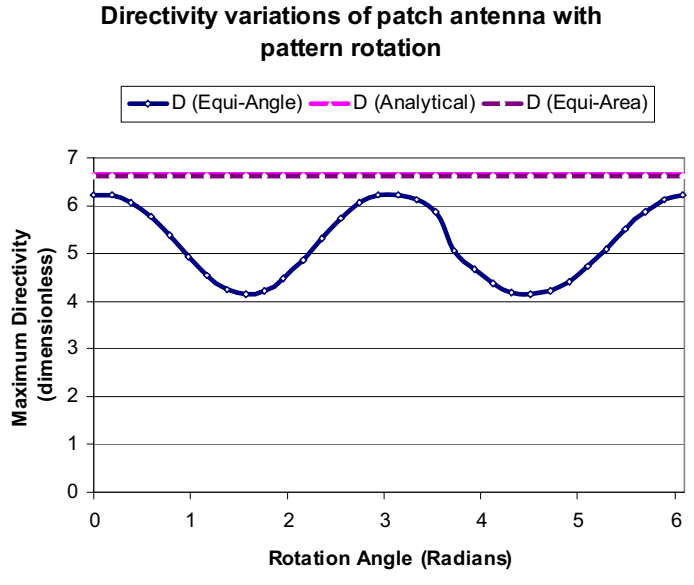

Fig. 3. Directivity variations of the patch antenna with changing rotation angle

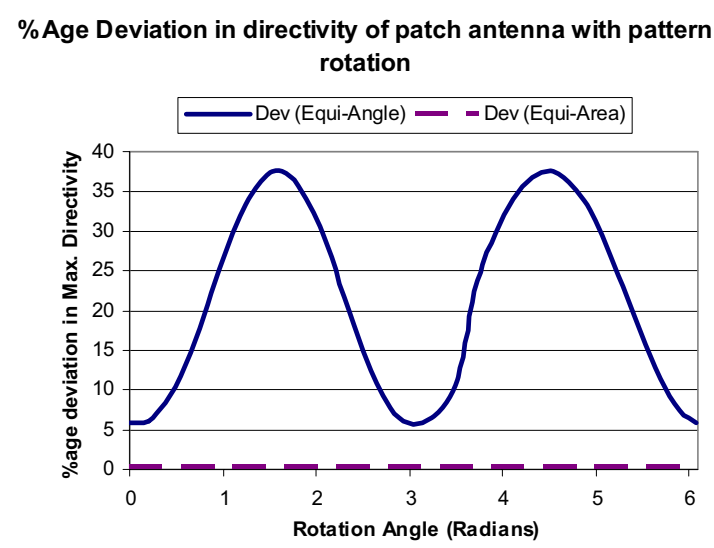

Fig. 4. \%Age deviation in directivity of the patch antenna

\section{Conclusion}

In this paper the equi-area-method is used to find the directivity of the patch with a rotating radiation pattern for 100 equi-area samples, and it is numerically demonstrated that the equi-area method gives more stable and accurate value of directivity as compared to the equi-angle based method (for 128 equi-angle samples). Consequently the equi-area method is independent of antenna boresight, and more efficient as it involves no weighting multiplications. This method is highly applicable to general antenna measurements, and can also be applied to determine the directivity of the high-gain beam-steered array antennas. Furthermore high sampling point densities on certain portions of the measurement sphere are avoided. The method also has a role in calculating and storing the patterns in a numerical simulation as it allows the radiation to be sampled accurately whilst using a smaller number of points than the equi-angle method.

\section{References}

[1] Michael D. Foegelle, “Antenna Pattern Measurement: Concepts and Techniques”, Compliance Engineering, Annual Reference Guide 2002.

[2] Constantine A. Balanis, “Antenna theory: analysis and design”, Wily, 1997.

[3] John. D. Kraus, “Antennas”, 2nd Ed., McGraw-Hill, Inc., 1988

[4] Paul Leopardi, "A partition of the unit sphere into regions of equal area and Small diameter", Electronic Transactions on Numerical Analysis, Volume 25, pp. 309-327, 2006. 